\title{
Critically ill patients with infectious diseases - clinical, evolutive and etiological issues
}

\author{
Irina Niculescu ${ }^{1,2^{*}}$, Augustin Cupşa ${ }^{1,2}$, Florentina Dumitrescu ${ }^{1,2}$, Iulian Diaconescu ${ }^{1,2}$, Livia Dragonu $u^{1,2}$, \\ Dan Hurezeanu ${ }^{1,2}$, Mariana Stănescu', Mihai Jianu $u^{1,2}$ \\ From The 10th Edition of the Scientific Days of the National Institute for Infectious Diseases "Prof Dr Matei \\ Bals" \\ Bucharest, Romania. 15-17 October 2014
}

\section{Background}

Infectious diseases (ID) are the second cause of death after cardiovascular diseases worldwide; severe sepsis and septic shock, syndromes induced by infection have an increasing incidence and high mortality requiring diagnosis and intensive therapy and appropriate attitude.

Objectives: to establish the clinical, evolutive and etiological aspects of ID evolving critically ill patients with ID.

\section{Methods}

Retrospective study (January 2013-March 2013) on 96 critically ill patients with ID hospitalized in the Intensive Care Unit of the "Victor Babeş" Hospital Craiova.

\section{Results}

General data of the study lot: gender distribution was balanced $(\mathrm{M}: \mathrm{F}=1: 1), 75 \%$ of patients were from urban areas and the median age was 60 (IQR: 1-95) years. ID were: respiratory infection $(67.71 \%)$, gastrointestinal (12.50\%), urinary $(9.37 \%)$, neurological $(9.37 \%)$ and cardiovascular (1.05\%). Failure or organ dysfunction (one or combination) in the study group: respiratory (69.79\%), neurological (9.37\%), renal (5.21\%), liver $(1.04 \%)$, hematologic $(1.04 \%)$ and heart (1.04\%). Sepsis was diagnosed in $35.42 \%$ of patients, severe sepsis in $22.92 \%$ and $5.21 \%$ developed septic shock.

Comorbidities were identified in $88.54 \%$ of patients (one or combination) as follows: chronic cardiovascular diseases $-39.58 \%$ of patients, neuropsychiatric $-16.67 \%$, pulmonary $-12.5 \%$, obesity $-10.42 \%$, HIV $-9.37 \%$, urogenital $-8.33 \%$, diabetes and cancer $-7.29 \%$ each, other
- $11.46 \%$. Favorable outcome was recorded in $88.54 \%$ of cases, death was recorded in $11.46 \%$ of cases. The etiology had been identified in $32.29 \%$ of the cases, as follows: bacterial etiology $-74.20 \%$ of the cases, viral and parasitic each in $12.90 \%$. The etiology of ID was represented by: Gram-negative bacteria (38.71\%), Gram-positive bacteria (22.58\%), Mycobacterium tuberculosis and Toxoplasma gondii (12.90\% in each case), measles virus (9.68\%) and pdm2009 influenza virus (3.23\%).

\section{Conclusion}

Critically ill patients with ID presented most commonly respiratory or gastrointestinal infection and most frequently developed respiratory or neurological failure or dysfunction. Most critically ill patients with ID associated comorbidities, most commonly chronic cardiovascular diseases. Evolving critically ill patients with ID had significantly increased risk of death. Gram-negative bacteria dominated the etiology of ID in critically ill patients, followed by Gram-positive bacteria.

\section{Authors' details \\ "Victor Babeş" Clinical Hospital of Infectious Diseases and Pneumology, Craiova, Romania. ${ }^{2}$ University of Medicine and Pharmacy Craiova, Romania.}

Published: 15 October 2014

doi:10.1186/1471-2334-14-S7-P22

Cite this article as: Niculescu et al: Critically ill patients with infectious diseases - clinical, evolutive and etiological issues. BMC Infectious Diseases 2014 14(Suppl 7):P22.

\footnotetext{
* Correspondence: iri_nic@yahoo.com

"Victor Babeș" Clinical Hospital of Infectious Diseases and Pneumology,

Craiova, Romania

Full list of author information is available at the end of the article
} 